\title{
Editorial
}

\section{Selected Papers from TimeNav 2007}

\author{
Patrizia Tavella ${ }^{1}$ and Demetrios Matsakis ${ }^{2}$ \\ ${ }^{1}$ Optics Department, Istituto Nazionale Ricerca Metrologica (INRIM), Strada delle Cacce 91, 10135 Torino, Italy \\ ${ }^{2}$ Time Service Department, US Naval Observatory, 3450 Massachusetts Avenue, NW, Washington, 20392-5420 DC, USA
}

Correspondence should be addressed to Patrizia Tavella, tavella@inrim.it

Received 26 June 2008; Accepted 26 June 2008

Copyright (C) 2008 P. Tavella and D. Matsakis. This is an open access article distributed under the Creative Commons Attribution License, which permits unrestricted use, distribution, and reproduction in any medium, provided the original work is properly cited.

As long as people have been navigating, they have needed to know how to measure time. Technology has changed the required level of precision, and the details of the application, but to-date some knowledge of time has always been required. In the earliest days, people would decide when to turn left or right by how many hours they had been walking or sailing. In the eighteenth century, contests were held and prizes awarded to those who could construct clocks accurate to the minute over monthly periods, so as to measure the longitude for navigational needs. And now, in the twenty-first century, we find navigation is dominated by radio-based global navigation satellite systems (GNSSs), which cannot function without knowledge of the time at the nanosecond level.

In April 2007, three important international meetings devoted to time, frequency, and navigation were held together in Geneva under a common umbrella aiming to promote the interchange of these communities. The meetings were

(i) the European Frequency and Time Forum (EFTF),

(ii) the IEEE Frequency Control Symposium (IEEEFCS),

(iii) the European Navigation Conference (ENC-GNSS).

With the development of the Galileo system, particular attention has arisen in Europe on research and advanced activities related to satellite navigation and its applications. Time and frequency are the heart of navigation systems given the "ticking" that synchronizes all the systems allowing positioning and also correct time dissemination.

The TimeNav event was therefore a unique event in relation to the IJNO aims of promoting and diffusing the culture of navigation. A special TimeNav issue focused on all the research themes related to the timing aspects of navigation systems was therefore proposed, and 11 authors accepted the invitation to submit a full paper describing their research activities relevant to the theme of time and navigation.

The resulting special issue that now is ready to appear is structured as follows. The first paper, by L. Galleani and P. Tavella, presents an application of the well-known Allan variance statistic, which is tailored to dynamic systems such as those relevant to navigation. There follow papers on how practical timekeeping is performed. One is from the USNO, which provides the time reference for GPS. Yuko Hanado et al. then describe the system at NICT, which is slated to provide the time reference for the QZSS system. The following three papers describe how GNSS control facilities generate time or ensure its validity. First, Carlos Hernández Medel et al. describe Galileo's integrity management, and then Qinghua Wang et al. provide an algorithm for backup control of the maser time reference. The third paper, by James R. Wright, describes how the GPS composite clock functions. Toshiaki Iwata et al. then show how a satellite clock can be synchronized. The following three papers, by Pascale Defraigne et al., J. Delporte et al., and G. Petit and Z. Jiang, complete the cycle by using the time of the GNSS systems to improve timekeeping on the ground. Finally, P. P. Sotiriadis and G. L. Weaver describe a new way of creating frequency synthesis which has the potential to greatly increase the available precision in future hardware.

Patrizia Tavella Demetrios Matsakis 

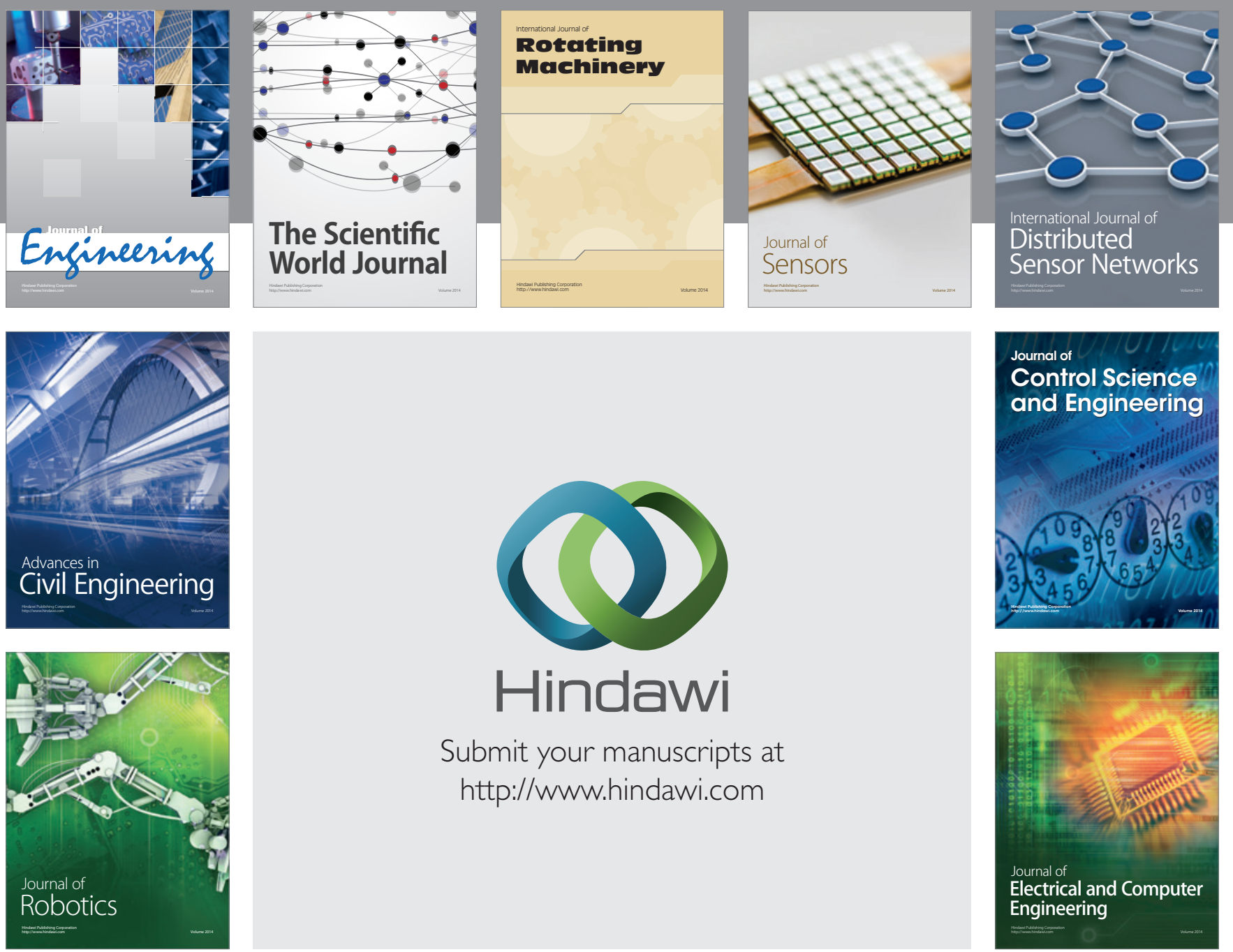

Submit your manuscripts at

http://www.hindawi.com
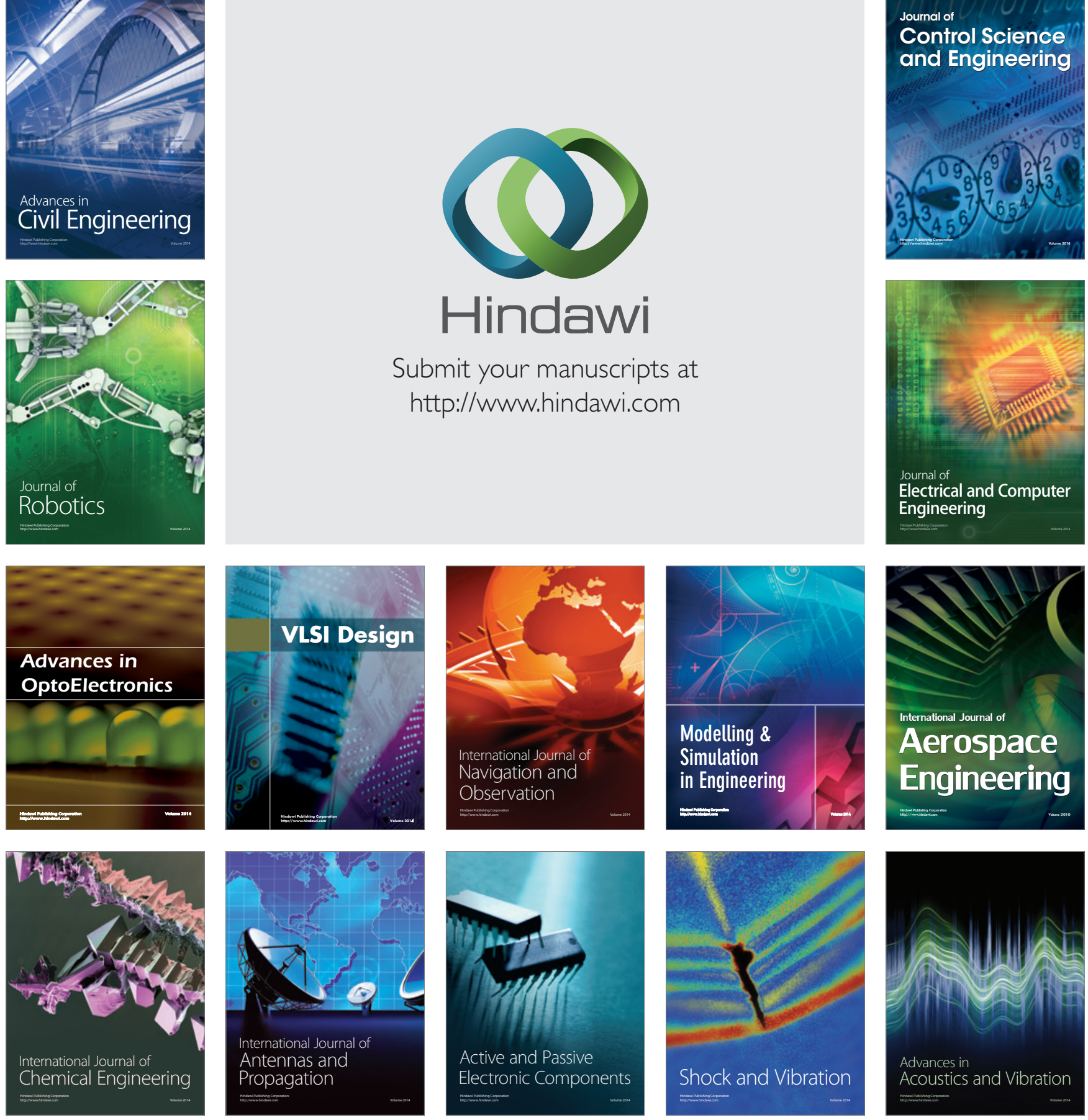